\title{
Bihamiltonian Equations on Polynomial Virasoro Algebras
}

Paolo CASATI ${ }^{a}$, Giovanni ORTENZI ${ }^{b}$

${ }^{a}$ Dipartimento di Matematica e applicazioni, II Università di Milano, Via R. Cozzi 53 Ed. U5, I-20126 Milano, Italy

E-mail: paolo.casati@unimib.it

${ }^{b}$ Dipartimento di Matematica, Politecnico di Torino, Corso Duca degli Abruzzi 24, I-10129 Torino, Italy

E-mail: giovanni.ortenzi@unimib.it

Received March 7, 2005; Accepted in Revised Form February 6, 2006

\begin{abstract}
We present and study bihamiltonian equations of Euler type which include a n-fields generalization of the Camassa-Holm equation living on the polynomial generalization of the Virasoro algebra possessing a wide number of independent central extensions.
\end{abstract}

\section{Introduction}

In a recent beautiful paper [6] Khesin and Misiołek have given a unified description as bihamiltonian systems of three non linear evolution equations living on the dual of the Virasoro algebra: the Korteweg-de Vries equation

$$
\frac{\partial u}{\partial t}=-3 \frac{\partial u}{\partial x} u+\frac{\partial^{3} u}{\partial x^{3}}
$$

the Camassa-Holm equation [2]

$$
\frac{\partial u}{\partial t}-\frac{\partial}{\partial t} \frac{\partial^{2} u}{\partial x^{2}}=-3 u \frac{\partial u}{\partial x}+2 \frac{\partial u}{\partial x} \frac{\partial^{2} u}{\partial x^{2}}+u \frac{\partial^{3} u}{\partial x^{3}}+c \frac{\partial^{3} u}{\partial x^{3}},
$$

and the Hunter-Saxon equation [4]

$$
\frac{\partial}{\partial t} \frac{\partial^{2} u}{\partial x^{2}}=-2 \frac{\partial u}{\partial x} \frac{\partial^{2} u}{\partial x^{2}}-u \frac{\partial^{3} u}{\partial x^{3}} .
$$

The key point in this unified description is that one of the two Poisson structures is the same for all the three equations and it is provided by the linear canonical Lie-Poisson bracket. While the other Poisson structure is constant and obtained by "freezing" the Lie-Poisson structure in a different point for each equation. Moreover all three equations are Euler equations with respect to three different Hamiltonians. 
The main purpose of this paper is to investigate if the recently appeared in the literature [10] "coupled" KdV equations living on the dual of polynomial extensions of the Virasoro Lie algebra:

$$
\alpha \frac{\partial u_{l}}{\partial t}=\sum_{j=0}^{l}\left(2 \alpha u_{j} \frac{\partial u_{l-j}}{\partial x}+\alpha \frac{\partial u_{j}}{\partial x} u_{l-j}-c_{j} \frac{\partial^{3} u_{l-j}}{\partial x^{3}}\right) \quad \text { for } l=0 \ldots n-1,
$$

share the same geometrical properties. More precisely we shall show that these latter equations have "bihamiltonian companions" which are "coupled" Camassa-Holm and HunterSaxon equations. Moreover it turns out that the more general Euler equation for the polynomial extensions of the Virasoro Lie algebra mixes up all the three cases. Finally it has be shown how in this general contest up to some minor technical requirements the bihamiltonian property of the equations still survives. Nevertheless we should point out that the interpretation of these coupled equations as geodesics of suitable Riemann metric is still an open question, being not yet clear if polynomial Virasoro algebras have or not a corresponding Lie group over them. However since a simliar problem arise when one consider the complex extension of the Virasoro algebra, it might be possible that such a Lie groups there exist for the real part of the polynomial Virasoro algebras.

The paper is organized as follows: in the next section we define a class of generalized Virasoro algebras, the polynomial ones and present their central extensions. The third section is devoted to compute the canonical Lie-Poisson brackets for these polynomial Virasoro algebras and the related Euler equations. In the last section we investigate their bihamiltonian formalism.

\section{Polynomial Virasoro algebras}

This first section is devoted to present some generalizations of the Virasoro algebras. These new algebras which we shall call "polynomial Virasoro algebras" to keep the nomenclature usually used in the literature for similar algebras [7], [1], are here defined as central extensions of tensor products among particular associative rings viewed as abelian Lie algebras and the Lie algebra $\operatorname{vect}\left(S^{1}\right)$ of smooth vector fields on the circle. More precisely let us denote as usual by $\mathbb{C}[\lambda]$ the associative ring of the complex polynomials in a complex variable $\lambda$. Since of course any subspace:

$$
\mathcal{I}_{n}[\lambda]=\left\{p(\lambda) \in \mathbb{C}[\lambda] \mid \exists q(\lambda) \in \mathbb{C}[\lambda]: p(\lambda)=\lambda^{n} q(\lambda)\right\}
$$

is an ideal in the commutative ring $\mathbb{C}[\lambda]$ we can consider the corresponding quotient rings.

Definition 2.1. Let $\mathbb{C}^{(n)}[\lambda]$ be the quotient polynomial ring

$$
\mathbb{C}^{(n)}[\lambda]=\mathbb{C}[\lambda] / \mathcal{I}_{n}(\lambda)
$$

where the quotient map $\Pi^{n}$ can be explicitly written as

$$
\begin{aligned}
\Pi^{n}: \mathbb{C}[\lambda] & \longrightarrow \mathbb{C}^{(n)}[\lambda] \\
\Pi^{n}\left(\sum_{j=0}^{\infty} p_{j} \lambda^{j}\right) & =\sum_{j=0}^{n-1} p_{j} \lambda^{j} .
\end{aligned}
$$


Now as already outlined above our "polynomial Virasoro algebras" will be defined by

Definition 2.2. Let vect ${ }^{(n)}\left(S^{1}\right)$ be the Lie algebra given by the tensor product

$$
\operatorname{vect}^{(n)}\left(S^{1}\right)=\operatorname{vect}\left(S^{1}\right) \otimes \mathbb{C}^{(n)}[\lambda] .
$$

Using the results of [1] and the well known fact that $\operatorname{vect}\left(S^{1}\right)$ is the Lie algebra of vector fields $u(x) \partial_{x}$ on the circle with Lie bracket (in what follows, to simplify the notations, we shall use $\partial_{x}^{s}$ instead of $\frac{\partial^{s}}{\partial x^{s}}$ with $s \in \mathbb{N}$ )

$$
\left[u(x) \partial_{x}, v(x) \partial_{x}\right]=-u(x) \partial_{x} v(x)+\left(\partial_{x} u(x)\right) v(x) \partial_{x}
$$

we may easily prove the following proposition which gives a suitable representation of the Lie algebra $\operatorname{vect}^{(n)}\left(S^{1}\right)$.

Proposition 2.3. The Lie algebra vect ${ }^{(n)}\left(S^{1}\right)$ of definition 2.3 is equivalent to the Lie algebra of "matrix" vector fields:

$$
u^{(n)}(x)=\left(\begin{array}{cccccc}
u_{0}(x) \partial_{x} & 0 & 0 & \ldots & 0 & 0 \\
u_{1}(x) \partial_{x} & u_{0}(x) \partial_{x} & 0 & \ldots & 0 & 0 \\
u_{2}(x) \partial_{x} & u_{1}(x) \partial_{x} & u_{0}(x) \partial_{x} & \ldots & 0 & 0 \\
\vdots & & & & & \vdots \\
u_{n-2}(x) \partial_{x} & u_{n-3}(x) \partial_{x} & u_{n-4}(x) \partial_{x} & \ldots & u_{0}(x) \partial_{x} & 0 \\
u_{n-1}(x) \partial_{x} & u_{n-2}(x) \partial_{x} & u_{n-1}(x) \partial_{x} & \ldots & u_{1}(x) \partial_{x} & u_{0}(x) \partial_{x}
\end{array}\right)
$$

and therefore its Lie bracket are given by

$$
\begin{aligned}
& {\left[u^{(n)}(x), v^{(n)}(x)\right]} \\
& =\left(\begin{array}{cccccc}
w_{0}(x) \partial_{x} & 0 & 0 & \ldots & 0 & 0 \\
w_{1}(x) \partial_{x} & w_{0}(x) \partial_{x} & 0 & \ldots & 0 & 0 \\
w_{2}(x) \partial_{x} & w_{1}(x) \partial_{x} & w_{0}(x) \partial_{x} & \ldots & 0 & 0 \\
\vdots & & & & & \vdots \\
w_{n-2}(x) \partial_{x} & w_{n-3}(x) \partial_{x} & w_{n-4}(x) \partial_{x} & \ldots & w_{0}(x) \partial_{x} & 0 \\
w_{n-1}(x) \partial_{x} & w_{n-2}(x) \partial_{x} & w_{n-3}(x) \partial_{x} & \ldots & w_{1}(x) \partial_{x} & w_{0}(x) \partial_{x}
\end{array}\right)
\end{aligned}
$$

where $w_{k}(x)$ is

$$
w_{k}(x)=\sum_{j=0}^{k}\left(-u_{j}(x) \partial_{x} v_{k-j}(x)+\left(\partial_{x} u_{j}(x)\right) v_{k-j}(x) \quad k=0, \ldots, n-1 .\right.
$$

Proof. In [1] has been proved that the map $\rho$ given by

$$
\begin{array}{ll}
\rho: \mathbb{C}^{(n)}[\lambda] \longrightarrow & \operatorname{End}\left(\mathbb{C}^{(n)}\right) \\
\rho\left(c_{i} \otimes \lambda^{i}\right) \longmapsto & c_{i} \Gamma^{i}
\end{array}
$$

where $\Gamma$ is the $n \times n$ matrix

$$
\Gamma=\sum_{i=0}^{n-1} e_{i+1, i}
$$


and

$$
\left(e_{i j}\right)_{k r}= \begin{cases}1 & \text { if } i=k, j=r \\ 0 & \text { otherwise }\end{cases}
$$

is a ring homomorphism. Therefore representing as usual [6], [8] the Lie algebra $\operatorname{vect}\left(S^{1}\right)$ as set of vector fields $u(x) \partial_{x}$ with Lie bracket 2.4 one gets immediately that two elements of vect ${ }^{(n)}\left(S^{1}\right)$ of the form $u_{j}(x) \partial_{x} \otimes \lambda^{j}$ and $v_{i}(x) \partial_{x} \otimes \lambda^{i}$ can be respectively represented in the form $\Gamma^{j} u_{j}(x) \partial_{x}$ and $\Gamma^{i} v_{i}(x) \partial_{x}$ with Lie bracket given by

$$
\left[\Gamma^{j} u(x)_{j} \partial_{x}, \Gamma^{i} v_{i}(x) \partial_{x}\right]=\left\{\begin{array}{l}
\Gamma^{i+j}\left(-u_{j}(x) \partial_{x} v(x)+\left(\partial_{x} u(x)\right) v(x)\right) \text { if } i+j \leq n-1 \\
0 \text { otherwise. }
\end{array}\right.
$$

Thus the statement of the proposition is obtained from these latter two equations by linear extension.

We should here remark that also the limit for $n$ goes to infinity of the Lie algebra $\operatorname{vect}\left(S^{1}\right) \otimes$ $\mathbb{C}^{\infty}[\lambda]$ make sense. The resulting algebra, which may be called vect ${ }^{(\infty)}\left(S^{1}\right)$, is obviously a subalgebra of loop algebra in $\operatorname{vect}\left(S^{1}\right)$, i.e. the algebra $\operatorname{vect}\left(S^{1}\right)$ which depends on one more parameter. More precisely vect ${ }^{(\infty)}\left(S^{1}\right)$ will be given by all the polynomial elements in the parameter $\lambda$ of the latter loop algebra. The generalization of the results presented in this paper to this more general setting and in particular the connection between this Lie algebra and the toroidal Lie algebras [9] are surely worth to be addressed in further publications. The authors warmly thank the anonymous referee who pointed have pointed out this aspect of the theory.

For $i=0, \ldots, n-1$ the Lie algebra vect ${ }^{(n)}\left(S^{1}\right)$ admits a $2-$ cocycle $C^{i}$. Indeed computations similar to those done for the usual Gelfand-Fuchs cocycle [3] show that the maps $C^{i}$ : $\operatorname{vect}^{(n)}\left(S^{1}\right) \times \operatorname{vect}^{(n)}\left(S^{1}\right) \rightarrow \mathbb{R}$ given by the formula:

$$
C^{i}\left(u^{(n)}, v^{(n)}\right)=\int_{S^{1}} \sum_{j=0}^{i} u_{j} \partial_{x}^{3} v_{i-j} \quad i=0, \ldots, n-1
$$

satisfy the 2 -cocycle conditions for vect ${ }^{(n)}\left(S^{1}\right)$. Since these cocycles are linear independent, for any subset $\left\{i_{0}, i_{1}, \ldots, i_{k}\right\}$ of $\{0,1, \ldots, n-1\}$ with $i_{j} \leq i_{k}$ if $j<k$, we can define $k+1$ dimensional extension of $\operatorname{vect}^{(n)}\left(S^{1}\right)$

Definition 2.4. For any subset non empty $\left\{i_{0}, i_{1}, \ldots, i_{k}\right\}$ of $\{0,1, \ldots, n-1\}$ with $i_{j} \leq i_{k}$ if $j<k$, let $\operatorname{vir}_{\left\{i_{0}, i_{1}, \ldots, i_{k}\right\}}^{(n)}$ denote the $k+1$-dimensional central extension of the Lie algebra vect $^{(n)}\left(S^{1}\right)$ :

$$
\operatorname{vir}_{\left\{i_{0}, i_{1}, \ldots, i_{k}\right\}}^{(n)}=\operatorname{vect}^{(n)}\left(S^{1}\right) \oplus \mathbb{R}^{k+1}
$$

with Lie brackets given by

$$
\begin{aligned}
& {\left[\left(v(x)^{(n)},\left(b_{i_{0}}, \ldots, b_{i_{k}}\right)\right),\left(u(x)^{(n)},\left(c_{i_{0}}, \ldots, c_{i_{k}}\right)\right)\right]=} \\
& \left(\left[v(x)^{(n)}, u(x)^{(n)}\right],\left(C^{i_{0}}\left(v(x)^{(n)}, u(x)^{(n)}\right), \ldots, C^{i_{k}}\left(v(x)^{(n)}, u(x)^{(n)}\right)\right)\right) .
\end{aligned}
$$




\section{Poisson framework for the Euler equations}

On each of the Lie algebras $\operatorname{vir}_{\left\{i_{0}, i_{1}, \ldots, i_{k}\right\}}^{(n)}$ is defined (see [1] for more details) a symmetric bilinear form $\langle\cdot, \cdot\rangle_{\left\{i_{0}, i_{1}, \ldots, i_{k}\right\}}^{(n)}$ given by the formula

$$
\begin{aligned}
& \left\langle u^{(n)}(x),\left(a_{i_{0}}, \ldots a_{i_{k}}\right),\left(v^{(n)}(x),\left(a_{i_{0}}, \ldots a_{i_{k}}\right)\right)\right\rangle_{\left\{i_{0}, i_{1}, \ldots, i_{k}\right\}}^{(n)} \\
& =\int_{S^{1}} \sum_{k=0}^{n-1}\left(\sum_{i=0}^{k} u_{i}(x) v_{k-i}(x)\right) d x+\sum_{j=0}^{n-1} a_{j} b_{n-j-1}
\end{aligned}
$$

where $a_{j}=b_{j}=0$ for $j \notin\left\{i_{0}, i_{1}, \ldots, i_{k}\right\}$. Let us denote by $\left(\operatorname{vir}_{\left\{i_{0}, i_{1}, \ldots, i_{k}\right\}}^{(n)}\right)^{*}$ the (geometrical) dual of $\operatorname{vir}_{\left\{i_{0}, i_{1}, \ldots, i_{k}\right\}}^{(n)}$ with respect to this pairing.

On it we can define the canonical Lie-Poisson structure as

$$
\{f, g\}(m)=\langle[d f, d g], m\rangle_{\left\{i_{0}, i_{1}, \ldots, i_{k}\right\}}^{(n)}
$$

for any $m \in\left(\operatorname{vir}_{\left\{i_{0}, i_{1}, \ldots, i_{k}\right\}}^{(n)}\right)^{*}$ and any two smooth functions $f, g$ on $\left(\operatorname{vir}_{\left\{i_{0}, i_{1}, \ldots, i_{k}\right\}}^{(n)}\right)^{*}$. (Here the differentials are taken at the point $m$ ).

By repeating the proof of Proposition 3.2 in [6] we can state the following

Proposition 3.1. The Poisson vector field on $\left(\operatorname{vir}_{\left\{i_{0}, i_{1}, \ldots, i_{k}\right\}}^{(n)}\right)^{*}$ corresponding to a Hamiltonian function $f$ and computed with respect to the canonical Lie-Poisson structure has the form:

$$
\frac{d m}{d t}=\left(a d_{\left\{i_{0}, i_{1}, \ldots, i_{k}\right\}}^{(n)}\right)_{d f}^{*} m
$$

where with $\left(a d_{\left\{i_{0}, i_{1}, \ldots, i_{k}\right\}}^{(n)}\right)^{*}$ we have denoted the coadjoint action of $\operatorname{vir}_{\left\{i_{0}, i_{1}, \ldots, i_{k}\right\}}^{(n)}$ on its dual.

Since this latter operator will play a crucial role in what follows let us compute it explicitly.

Proposition 3.2. Let $\left(u^{(n)}, \underline{a}\right)$ and $\left(m^{(n)}, \underline{c}\right)$ elements of $\operatorname{vir}_{\left\{i_{0}, i_{1}, \ldots, i_{k}\right\}}^{(n)}$ and $\left(\operatorname{vir}_{\left\{i_{0}, i_{1}, \ldots, i_{k}\right\}}^{(n)}\right)^{*}$ respectively (where to keep short the notations we regard $\underline{b}$ and $\underline{c}$ as vectors in $\mathbb{R}^{n}$ with $b_{i_{r}}=c_{i_{r}}=0$ if $\left.i_{r} \notin\left\{i_{0}, \ldots, i_{k}\right\}\right)$ then

$$
\left.\begin{array}{rl}
\left(a d_{\left\{i_{0}, i_{1}, \ldots, i_{k}\right\}}^{(n)}\right)_{\left(u^{(n)}, \underline{a}\right)}^{*}\left(m^{(n)}, \underline{c}\right) \\
2 m_{0} \partial_{x} u_{0}+\left(\partial_{x} m_{0}\right) u_{0}-c_{0} \partial_{x}^{3} u_{0} \\
2 m_{1} \partial_{x} u_{0}+\left(\partial_{x} m_{1}\right) u_{0}+2 m_{0} \partial_{x} u_{1} \\
+\left(\partial_{x} m_{0}\right) u_{1}-c_{0} \partial_{x}^{3} u_{1}-c_{1} \partial_{x}^{3} u_{0} \\
\vdots \\
\vdots \\
\sum_{j=0}^{l}\left(2 m_{j} \partial_{x} u_{l-j}+\left(\partial_{x} m_{j}\right) u_{l-j}-c_{j} \partial_{x}^{3} u_{l-j}\right) \\
\vdots \\
\sum_{j=0}^{n-1}\left(2 m_{j} \partial_{x} u_{n-j-1}+\left(\partial_{x} m_{j}\right) u_{n-j-1}-c_{j} \partial_{x}^{3} u_{n-j-1}\right)
\end{array}\right), \underline{0}
$$


Proof. Let $\left(m^{(n)}(x),\left(c_{i_{0}}, \ldots, c_{i_{k}}\right)\right)=\left(m^{(n)}, \underline{c}\right)$ a generic element in $\left(\operatorname{vir}_{\left\{i_{0}, i_{1}, \ldots, i_{k}\right\}}^{(n)}\right)^{*}$ then for every $\left(v^{(n)}, \underline{b}\right)$ in $\operatorname{vir}_{\left\{i_{0}, i_{1}, \ldots, i_{k}\right\}}^{(n)}$ by definition we have

$$
\begin{aligned}
& \left(\left\langle v^{(n)}(x), \underline{b}\right),\left(\operatorname{ad}_{\left\{i_{0}, i_{1}, \ldots, i_{k}\right\}}^{(n)}\right)_{\left(u^{(n)}, \underline{a}\right)}^{*}\left(m^{(n)}, \underline{c}\right)\right\rangle_{\left\{i_{0}, i_{1}, \ldots, i_{k}\right\}}^{(n)} \\
& =\left\langle\left(\operatorname{ad}_{\left\{i_{0}, i_{1}, \ldots, i_{k}\right\}}^{(n)}\right)_{\left(u^{(n)}, \underline{a}\right)}\left(v^{(n)}, \underline{b}\right),\left(m^{(n)}, \underline{c}\right)\right\rangle_{\left\{i_{0}, i_{1}, \ldots, i_{k}\right\}}^{(n)} .
\end{aligned}
$$

Explicitly recalling definition 2.4 proposition 2.6 and changing the order in the sums we have

$$
\begin{aligned}
& \left\langle\left(\operatorname{ad}_{\left\{i_{0}, i_{1}, \ldots, i_{k}\right\}}^{(n)}\right)_{\left(u^{(n)}, \underline{a}\right)}\left(v^{(n)}\right),\left(m^{(n)}, \underline{c}\right)\right\rangle_{\left\{i_{0}, i_{1}, \ldots, i_{k}\right\}}^{(n)} \\
& =\int_{S^{1}}\left\{\sum_{k=0}^{n-1} \sum_{i=0}^{k}\left(\sum_{j=0}^{i}\left(-u_{j} \partial_{x} v_{i-j}+\left(\partial_{x} u_{j}\right) v_{i-j}\right) m_{k-i}\right)\right. \\
& \left.+\sum_{k=0}^{n-1} \sum_{i=0}^{k}\left(\sum_{s=0}^{i}\left(u_{s} \partial_{x}^{3} v_{i-s}\right) c_{k-i}\right)\right\} d x \\
& =\int_{S^{1}}\left\{\sum_{k=0}^{n-1} \sum_{i=0}^{k} \sum_{j=0}^{i}\left(2 u_{j} \partial_{x} m_{k-i}+\left(\partial_{x} u_{j}\right) m_{k-i}-c_{k-i} \partial_{x}^{3} u_{j}\right) v_{i-j}\right\} d x \\
& =\int_{S^{1}}\left\{\sum_{k=0}^{n-1} \sum_{i=0}^{k} \sum_{j=0}^{i}\left(2 u_{j} \partial_{x} m_{i-j}+\left(\partial_{x} u_{j}\right) m_{i-j}-c_{i-j} \partial_{x}^{3} u_{j}\right) v_{k-i}\right\} d x \\
& =\sum_{i=0}^{n-1} \sum_{j=0}^{i} \int_{S^{1}}\left(\left(\operatorname{ad}_{\left\{i_{0}, i_{1}, \ldots, i_{k}\right\}}^{(n)}\right)_{\left(u^{(n)}, \underline{a}\right)}^{*}\left(m^{(n)}, \underline{c}\right)\right)_{i} v_{i-j} d x \\
& =\left\langle\left(v^{(n)}, \underline{a}\right),\left(\operatorname{ad}_{\left\{i_{0}, i_{1}, \ldots, i_{k}\right\}}^{(n)}\right)_{\left(u^{(n)}, \underline{a}\right)}^{*},\left(m^{(n)}, \underline{c}\right)\right\rangle \text {. }
\end{aligned}
$$

From the above computation we have that the l-th component of the co-adjoint action of $\left(u^{(n)}, \underline{b}\right)$ evaluated in a generic vector $\left(m^{(n)}, \underline{c}\right)$ is:

$$
\left(\left(\operatorname{ad}_{\left\{i_{0}, i_{1}, \ldots, i_{k}\right\}}^{(n)}\right)_{\left(u^{(n)}, \underline{a}\right)}^{*}\left(m^{(n)}, \underline{c}\right)\right)_{l}=\left(\sum_{j=0}^{l} 2 m_{j} \partial_{x} u_{l-j}+\left(\partial_{x} m_{j}\right) u_{l-j}-c_{j} \partial_{x}^{3} u_{l-j}, 0\right) .
$$

This proves the statement of the proposition.

Using equation (3.2) we can canonically associate a Poisson structure with this coadjoint action. It holds indeed:

Proposition 3.3. For all $\left(m^{(n)}, \underline{c}\right) \in\left(\operatorname{vir}_{\left\{i_{0} \ldots i_{k}\right\}}^{(n)}\right)^{*}$, the operator :

$$
\begin{aligned}
& P(m): \operatorname{vir}_{\left\{i_{0} \ldots i_{k}\right\}}^{(n)} \rightarrow\left(\operatorname{vir}_{\left\{i_{0} \ldots i_{k}\right\}}^{(n)}\right)^{*} \\
& P(m)=\left(\begin{array}{ccccccc}
P_{0}(m) & 0 & 0 & 0 & \ldots & 0 & 0 \\
P_{1}(m) & P_{0}(m) & 0 & 0 & \ldots & 0 & 0 \\
P_{2}(m) & P_{1}(m) & P_{0}(m) & 0 & \ldots & 0 & 0 \\
\vdots & \ddots & \ddots & \ddots & \ldots & 0 & 0 \\
P_{l}(m) & \vdots & \ddots & \ddots & \ddots & \ldots & 0 \\
\vdots & \vdots & \vdots & \ddots & \ddots & \ddots & \vdots \\
P_{n-1}(m) & P_{n-2}(m) & \ldots & \ldots & P_{2}(m) & P_{1}(m) & P_{0}(m)
\end{array}\right)
\end{aligned}
$$

where $m=\left(m^{(n)}, \underline{c}\right)$ and $P_{l}(m):=c_{l} \partial_{x}^{3}+\left(m_{l} \partial_{x}+\partial_{x} m_{l}\right)$, defines a Poisson structure:

$$
\left\{\left(u^{(n)}, \underline{a}\right),\left(v^{(n)}, \underline{b}\right)\right\}(m)=\left\langle\left(v^{(n)}, \underline{b}\right), P(m)\left(u^{(n)}, \underline{a}\right)\right\rangle_{\left\{i_{0}, \ldots, i_{k}\right\}}^{(n)}
$$


Proof. It is a simple computation to shows that

$$
\left\langle\left(v^{(n)}, \underline{b}\right), P(m)\left(u^{(n)}, \underline{a}\right)\right\rangle_{\left\{i_{0}, \ldots, i_{k}\right\}}^{(n)}=\left\langle\left(v^{(n)}, \underline{b}\right),\left(\operatorname{ad}_{\left\{i_{0}, \ldots, i_{k}\right\}}^{(n)}\right)_{\left(u^{(n)}, \underline{a}\right)}^{*}\left(m^{(n)}, \underline{c}\right)\right\rangle_{\left\{i_{0}, \ldots, i_{k}\right\}}^{(n)}
$$

follows directly from the previous proposition.

Observe that the operator (3.4) is not apparently antisymmetric because the inner product (3.1) is not diagonal.

We want now use the Poisson tensor (3.4) in order to define the Euler equation on $\left(\operatorname{vir}_{\left\{i_{0}, i_{1}, \ldots, i_{k}\right\}}^{(n)}\right)^{*}$. Following [6] we may define the following $2(n+1)$-parameter family of quadratic forms " $H_{\underline{\alpha}, \underline{\beta}}^{1}$ energies" $\left(\underline{\alpha}=\left(\alpha_{0}, \ldots, \alpha_{n}\right), \underline{\beta}=\left(\beta_{0}, \ldots, \beta_{n}\right)\right)$ on the Lie algebra $\operatorname{vir}_{\left\{i_{0}, i_{1}, \ldots, i_{k}\right\}}^{(n)}$ :

$$
\begin{aligned}
& \left.\left\langle\left(v^{(n)}(x),\left(b_{i_{0}}, \ldots, b_{i_{k}}\right)\right),\left(w^{(n)}(x), c_{i_{0}}, \ldots, c_{i_{k}}\right)\right)\right\rangle_{H_{\underline{\alpha}, \underline{\beta}}^{1}} \\
& =\int_{S^{1}}\left(\sum_{i=0}^{n-1}\left(\sum_{j=0}^{i} \alpha_{i} v_{j} w_{i-j}+\sum_{j=0}^{i} \beta_{i}\left(\partial_{x} v_{j}\right) \partial_{x} w_{i-j}\right) d x+\sum_{j=0}^{n} b_{j} c_{n-j-1} .\right.
\end{aligned}
$$

where $c_{i_{r}}=b_{i_{r}}=0$ if $i_{k} \notin\left\{i_{0}, i_{1}, \ldots, i_{k}\right\}$.

Of course the case $\alpha_{i}=1$ and $\beta_{i}=0(i=0, \ldots, n)$ correspond to a $L^{2}$ inner product while the case $\alpha_{i}=1 \beta_{i}=1(i=0, \ldots, n)$ to a $H^{1}$-like one. With each energy form $H_{\underline{\alpha}, \underline{\beta}}^{1}(3.5)$ is associated a corresponding "inertia operator":

$$
A^{\underline{\alpha}, \underline{\beta}}:\left(\operatorname{vir}_{\left\{i_{0}, i_{1}, \ldots, i_{k}\right\}}^{(n)}\right) \rightarrow\left(\operatorname{vir}_{\left\{i_{0}, i_{1}, \ldots, i_{k}\right\}}^{(n)}\right)^{*}
$$

defined by the equation

$$
\begin{aligned}
& \left.\left\langle\left(v^{(n)}(x),\left(b_{i_{0}}, \ldots, b_{i_{k}}\right)\right), A^{\underline{\alpha}, \underline{\beta}}\left(w^{(n)}(x), c_{i_{0}}, \ldots, c_{i_{k}}\right)\right)\right\rangle_{\left\{i_{0}, \ldots, i_{k}\right\}}^{(n)} \\
& \left.=\left\langle\left(v^{(n)}(x),\left(b_{i_{0}}, \ldots, b_{i_{k}}\right)\right),\left(w^{(n)}(x), c_{i_{0}}, \ldots, c_{i_{k}}\right)\right)\right\rangle_{H_{\underline{\alpha}, \underline{\beta}}^{1}}^{1}
\end{aligned}
$$

so that the $H_{\underline{\alpha}, \underline{\beta}}^{1}$-energy is given by the formula

$$
E_{\underline{\alpha}, \underline{\beta}}(v)=\frac{1}{2}\left\langle\left(v^{(n)}(x),\left(b_{i_{0}}, \ldots, b_{i_{k}}\right)\right),\left(A^{\underline{\alpha}, \underline{\beta}}\left(v^{(n)}(x), b_{i_{0}}, \ldots, b_{i_{k}}\right)\right)\right\rangle_{\left\{i_{0}, \ldots, i_{k}\right\}}^{(n)} .
$$

From equation (3.7) we get explicitly

Proposition 3.4. The explicit form of the "inertia operator" $A^{\underline{\alpha}, \underline{\beta}}$ of $\operatorname{vir}_{\left\{i_{0}, i_{1}, \ldots, i_{k}\right\}}^{(n)}$ is

$$
A^{\underline{\alpha}, \underline{\beta}}=\left(\begin{array}{ccccccc}
\Lambda_{n-1} & 0 & 0 & 0 & \ldots & 0 & 0 \\
\Lambda_{n-2}-\Lambda_{n-1} & \Lambda_{n-1} & 0 & 0 & \ldots & 0 & 0 \\
\Lambda_{n-3}-\Lambda_{n-2} & \Lambda_{n-2}-\Lambda_{n-1} & \Lambda_{n-1} & 0 & \ldots & 0 & 0 \\
\vdots & \ddots & \ddots & \ddots & \ldots & 0 & 0 \\
\Lambda_{n-i+1}-\Lambda_{n-i} & \vdots & \ddots & \ddots & \Lambda_{n-1} & \ldots & 0 \\
\vdots & \vdots & \vdots & \ddots & \ddots & \ddots & \vdots \\
\Lambda_{0}-\Lambda_{1} & \Lambda_{1}-\Lambda_{2} & \ldots & \ldots & \Lambda_{n-3}-\Lambda_{n-2} & \Lambda_{n-2}-\Lambda_{n-1} & \Lambda_{n-1}
\end{array}\right)
$$


with $\Lambda_{i}=\alpha_{i}-\beta_{i} \partial_{x}^{2}$ i.e.:

$$
\begin{aligned}
A_{i, \underline{\alpha}}^{\underline{\alpha}, \underline{\beta}}=0 & & \text { if } j>i, i, j=0, \ldots, n-1 \\
A_{i, \underline{\alpha}}^{\underline{\alpha}, \underline{\beta}}=\alpha_{n-1}-\beta_{n-1} \partial_{x}^{2} & & \text { if } i=0, \ldots, n-1 \\
A_{i, \underline{\underline{\alpha}}}^{\underline{\alpha} \underline{\underline{\beta}}}=\alpha_{n-i+j+1}-\alpha_{n-i+j}-\left(\beta_{n-i+j+1}-\beta_{n-i+j}\right) \partial_{x}^{2} & & \text { if } i=1, \ldots, n-1 \\
& & j=0, \ldots, i-1 .
\end{aligned}
$$

Proof. By expanding in components equation (3.7) and by subtracting the contribute due to the central extension we obtain

$$
\int_{S^{1}} \sum_{j=0}^{n-1} v_{j} \sum_{k=0}^{n-1} w_{k} \sum_{i=0}^{n-j-1} A_{i, k}^{\underline{\alpha}}, \underline{\beta} d x=\int_{S^{1}} \sum_{s=0}^{n-1} \alpha_{s} \sum_{r=0}^{n-s-1} v_{r} w_{r-s}+\sum_{s=0}^{n-1} \beta_{s} \sum_{r=0}^{n-s-1}\left(\partial_{x} v_{r}\right) \partial_{x} w_{r-s} d x .
$$

Then operating by part on the second hand of this equation we obtain the following $n^{2}$ equations on the entries of $A^{\underline{\alpha}, \underline{\beta}}$ :

$$
\sum_{i=0}^{n-j-1} A_{i, k}^{\underline{\alpha}, \underline{\beta}}=\gamma_{i, k} \quad j, k=0, \ldots n-1,
$$

where

$$
\gamma_{j, k}= \begin{cases}0 & \text { if } j+k>n-1 \\ \alpha_{s}-\beta_{s} \partial_{x}^{2} & \text { if } j+k=s \leq n-1 .\end{cases}
$$

Since this latter system is lower triangular, it admits the unique solution:

$$
\begin{array}{rlrl}
A_{i, \underline{\alpha}}^{\underline{\alpha}, \underline{\beta}}=0 & \text { if } j>i, i, j=0, \ldots, n-1 \\
A_{i, i}^{\underline{\alpha}, \underline{\beta}}=\alpha_{n-1}-\beta_{n-1} \partial_{x}^{2} & \text { if } i=0, \ldots, n-1 \\
A_{i, \underline{\underline{\alpha}}}^{\underline{\underline{\alpha}}, \underline{\beta}}=\alpha_{n-i+j+1}-\alpha_{n-i+j}-\left(\beta_{n-i+j+1}-\beta_{n-i+j}\right) \partial_{x}^{2} & \text { if } i=1, \ldots, n-1 \\
& & j=0, \ldots, i-1 .
\end{array}
$$

Using the previous construction we may consider the following Euler equations on $\left(\operatorname{vir}_{\left\{i_{0}, \ldots, i_{k}\right\}}^{(n)}\right)^{*}$ :

$$
\frac{d\left(A^{\underline{\alpha}, \underline{\beta}} u\right)}{d t}=\left(\operatorname{ad}_{\left\{i_{0}, i_{1}, \ldots, i_{k}\right\}}^{(n)}\right)_{u}^{*} A^{\underline{\alpha}, \underline{\beta}} u=P\left(A^{\underline{\alpha}, \underline{\beta}} u\right) u
$$

which written explicitly in components becomes

$$
\begin{aligned}
& \sum_{r=l}^{1}\left[\left(\alpha_{n-r}-\alpha_{n-r+1}\right) \partial_{t} u_{l-r}-\left(\beta_{n-r}-\beta_{n-r+1}\right) \partial_{t} \partial_{x}^{2} u_{l-r}\right]+ \\
& +\alpha_{n} \partial_{t} u_{l}-\beta_{n} \partial_{t} \partial_{x}^{2} u_{l}= \\
& \sum_{j=0}^{l}\left\{2 \left[\sum_{r=1}^{j}\left(\left(\alpha_{n-r}-\alpha_{n-r+1}\right) u_{j-r}-\left(\beta_{n-r}-\beta_{n-r+1}\right) \partial_{x}^{2} u_{j-r}\right)+\right.\right. \\
& \left.\left.+\alpha_{n} u_{j}-\beta_{n} \partial_{x}^{2} u_{j}\right)\right] \partial_{x} u_{l-j}+\left[\sum _ { r = 1 } ^ { j } \left(\left(\alpha_{n-r}-\alpha_{n-r+1}\right) \partial_{x} u_{j-r}+\right.\right. \\
& \left.\left.\left.-\left(\beta_{n-r}-\beta_{n-r+1}\right) \partial_{x}^{3} u_{j-r}\right)+\alpha_{n} \partial_{x} u_{j}-\beta_{n} \partial_{x}^{3} u_{j}\right] u_{l-j}-c_{j} \partial_{x}^{3} u_{l-j}\right\}
\end{aligned}
$$

for $l=0 \ldots n-1$. 
Remark 3.5. When $\frac{\alpha_{i}}{\alpha_{n-1}}=\frac{\beta_{i}}{\beta_{n-1}}=1$ for $i=0 \ldots n-1$, i.e. $\Lambda_{i}=\Lambda=\alpha_{n-1}-\beta_{n-1} \partial_{x}^{2} \quad i=$ $0 \ldots n-1$ and therefore the "inertia operator" is diagonal, the system (3.8) becomes:

$$
\Lambda \partial_{t} u_{l}=\sum_{j=0}^{l}\left[2\left(\Lambda u_{j}\right) \partial_{x} u_{l-j}+\left(\Lambda \partial_{x} u_{j}\right) u_{l-j}+c_{j} \partial_{x}^{3} u_{l-j}\right]
$$

for $l=0 \ldots n-1$.

Let us end this section by writing down explicitly the first two non trivial cases of equation (3.8), namely the case $n=2, n=3$ respectively:

$$
\begin{gathered}
\left\{\begin{array}{l}
\partial_{t} \alpha_{1} u_{0}-\beta_{1} \partial_{t} \partial_{x}^{2} u_{0}=3 \alpha_{1}\left(\partial_{x} u_{0}\right) u_{0}-2 \beta_{1}\left(\partial_{x}^{2} u_{0}\right) \partial_{x} u_{0}-\beta_{1}\left(\partial_{x}^{3} u_{0}\right) u_{0}-c_{0} \partial_{x}^{3} u_{0} \\
\alpha_{0} \partial_{t} u_{0}-\beta_{0} \partial_{t} \partial_{x}^{2} u_{0}+\alpha_{1} \partial_{t} u_{1}-\beta_{1} \partial_{t} \partial_{x}^{2} u_{1} \\
=3 \alpha_{0}\left(\partial_{x} u_{0}\right) u_{0}-2 \beta_{0}\left(\partial_{x}^{2} u_{0}\right) \partial_{x} u_{0}-\beta_{0}\left(\partial_{x}^{3} u_{0}\right) u_{0}-\left(c_{1}+c_{0}\right) \partial_{x}^{3} u_{0}+ \\
+3 \alpha_{1} \partial_{x}\left(u_{0} u_{1}\right)-2 \beta_{1} \partial_{x}\left(\left(\partial_{x} u_{0}\right) \partial_{x} u_{1}\right)-\beta_{1}\left(\partial_{x}^{3} u_{1}\right) u_{0}-\beta_{1}\left(\partial_{x}^{3} u_{0}\right) u_{1}-c_{0} \partial_{x}^{3} u_{1}
\end{array}\right. \\
\left\{\begin{array}{l}
\left.\alpha_{2} \partial_{t} u_{0}-\beta_{2} \partial_{t} \partial_{x}^{2} u_{0}=3 \alpha_{2}\left(\partial_{x} u_{0}\right) u_{0}-2 \beta_{2}\left(\partial_{x}^{2} u_{0}\right) \partial_{x} u_{0}\right)-\beta_{2}\left(\partial_{x}^{3} u_{0}\right) u_{0}-c_{0} \partial_{x}^{3} u_{0} \\
\alpha_{1} \partial_{t} u_{0}-\beta_{1} \partial_{t} \partial_{x}^{2} u_{0}+\alpha_{2} \partial_{t} u_{1}-\beta_{2} \partial_{t} \partial_{x}^{2} u_{1}= \\
=3 \alpha_{1}\left(\partial_{x} u_{0}\right) u_{0}-2 \beta_{1}\left(\partial_{x}^{2} u_{0}\right) \partial_{x} u_{0}-\beta_{1}\left(\partial_{x}^{3} u_{0}\right) u_{0}-\left(c_{1}+c_{0}\right) \partial_{x}^{3} u_{0}+ \\
+3 \alpha_{2} \partial_{x}\left(u_{0} u_{1}\right)-2 \beta_{2} \partial_{x}\left(\left(\partial_{x} u_{0}\right) \partial_{x} u_{1}\right)-\beta_{2}\left(\partial_{x}^{3} u_{1}\right) u_{0}-\beta_{2}\left(\partial_{x}^{3} u_{0}\right) u_{1}-c_{0} \partial_{x}^{3} u_{1} \\
\alpha_{0} \partial_{t} u_{0}-\beta_{0} \partial_{t} \partial_{x}^{2} u_{0}+\alpha_{1} \partial_{t} u_{1}-\beta_{1} \partial_{t} \partial_{x}^{2} u_{1}+\alpha_{2} \partial_{t} u_{2}-\beta_{2} \partial_{t} \partial_{x}^{2} u_{2} \\
=3 \alpha_{0}\left(\partial_{x} u_{0}\right) u_{0}-2 \beta_{0}\left(\partial_{x}^{2} u_{0}\right) \partial_{x} u_{0}-\beta_{0}\left(\partial_{x}^{3} u_{0}\right) u_{0}-\left(c_{2}+c_{1}+c_{0}\right) \partial_{x}^{3} u_{0} \\
+3 \alpha_{1} \partial_{x}\left(u_{0} u_{1}\right)-2 \beta_{1} \partial_{x}\left(\left(\partial_{x} u_{0}\right) \partial_{x} u_{1}\right)-\beta_{1}\left(\partial_{x}^{3} u_{1}\right) u_{0}-\beta_{1}\left(\partial_{x}^{3} u_{0}\right) u_{1} \\
-\left(c_{1}+c_{0}\right) \partial_{x}^{3} u_{1}+3 \alpha_{2} \partial_{x}\left(u_{0} u_{2}\right)+3 \alpha_{2} u_{1} \partial_{x} u_{1}-2 \beta_{2} \partial_{x}\left(\left(\partial_{x} u_{0}\right) \partial_{x} u_{2}\right) \\
-2 \beta_{2}\left(\partial_{x}^{2} u_{1}\right) \partial_{x} u_{1}-\beta_{2}\left(\partial_{x}^{3} u_{0}\right) u_{2}-\beta_{2}\left(\partial_{x}^{3} u_{2}\right) u_{0}-\beta_{2}\left(\partial_{x}^{3} u_{1}\right) u_{1}-c_{0} \partial_{x}^{3} u_{2}
\end{array}\right.
\end{gathered}
$$

\section{Bihamiltonian setting}

The equations written in the previous section reduce in the case of $n=0$ to the $\mathrm{KdV}$ the Hunter-Saxon and the Camassa-Holm (where $\alpha_{i} \neq 0, \beta_{i}=0$ for $\mathrm{KdV}$, and $\alpha_{i}=0$, $\beta_{i} \neq 0$ for Hunter-Saxon, and finally both $\alpha_{i} \neq 0, \beta_{i} \neq 0$ for Camassa-Holm). These latter equations own all a bihamiltonian structure (see for example [6] or Camassa Holm [2]). This suggests to look for a similar bihamiltonian structure in the general case. Unfortunately in this more general contest, when we have to deal with $n>0$ the things become quite messy. Therefore to make the construction more transparent let us tackle the most simple case when the inertial operator $A^{\underline{\alpha}, \underline{\beta}}$ of proposition 3.4 is written in diagonal form, i.e., when the coefficients $\alpha_{i}$ and $\beta_{i}$ appearing in the formula of the energy (3.5) satisfy the relations $\alpha_{i}=\alpha_{j}=\alpha$ and $\beta_{i}=\beta_{j}=\beta$ for all $i, j=0, \ldots, n-1$ and $c_{i}=\delta_{0 i}$ $(i=0, \ldots, n-1)$; in spite of the fact that we are confident that up to some very involute computations the same results should still hold in a more general setting. In this particular case the Euler equations become (3.9). For these equations when $\alpha \neq 0$ it is easy to prove the following 
Theorem 4.1. The equations (3.9) for $\alpha \neq 0$ are bihamiltonian on vir ${ }^{(n)}$ with Hamiltonians given by

$$
\begin{aligned}
H= & \int_{S^{1}}\left(\sum _ { l = 0 } ^ { n - 1 } \left(\sum_{\substack{i, j, k=0 \\
i+j+k=n-1}}^{l} \frac{\alpha}{4}\left(\Lambda^{-1}\left(u_{i}\right) \Lambda^{-1}\left(u_{j}\right) \Lambda^{-1}\left(u_{k}\right)+\frac{1}{4} \Lambda^{-1}\left(u_{i}\right) \Lambda^{-1}\left(u_{j}\right) u_{k}\right)\right.\right. \\
& \left.+\frac{a}{2} \sum_{\substack{i, j=0 \\
i+j=n-1}}^{n-1} \Lambda^{-1}\left(\partial_{x} u_{i}\right) \Lambda^{-1}\left(\partial_{x} u_{j}\right)\right) d x=\int_{S^{1}} h d x
\end{aligned}
$$

where $\Lambda=\alpha-\beta \partial_{x}^{2}$ and with second Poisson tensor is obtained freezing the canonical one at the point $u_{0}=\frac{1}{2}, u_{j}=0$ for all $j=1, \ldots, n-1$.

Proof. Let us first compute the variational derivatives with respect to the variables $u_{j}$ $\frac{\delta h}{\delta u_{0}}, \ldots \frac{\delta h}{\delta u_{n-1}}$ of the functional $h$, because those corresponding to the variables in the central extension do not play any role in what follows. This amounts to determine the gradient $\left(h_{0}, \ldots, h_{n-1}\right)$ in $\left(\operatorname{vir}_{i_{0}, \ldots, i_{k}}^{(n)}\right)^{*}$ of the functional $h$ with respect to these variables which is defined by the following identities holding for any vector $\underline{\xi}=\left(\xi_{0}, \ldots, \xi_{n-1}\right)$ in $\operatorname{vir}_{i_{0}, \ldots, i_{k}}^{(n)}$ :

$$
\left\langle\left(\xi_{0}, \ldots, \xi_{n-1}\right),\left(h_{0}, \ldots, h_{n-1}\right)\right\rangle_{i_{0}, \ldots, i_{k}}^{(n)}=\left.\frac{d h\left(u_{0}+\epsilon \xi_{0}, \ldots, u_{n-1}+\epsilon \xi_{n-1}\right)}{d \epsilon}\right|_{\epsilon=0} .
$$

From the definition of our bilinear form (3.1) this equation boils down to the relation

$$
\sum_{k=0}^{n-1} \sum_{i=0}^{k} \xi_{i} h_{k-i}=\sum_{k=0}^{n-1} \frac{\delta h}{\delta u_{k}} \xi_{k}
$$

where $\frac{\delta h}{\delta u_{i}}$ is given by the usual formula $\frac{\delta h}{\delta u_{i}}=\sum_{s=0}^{\infty}\left(-\partial_{x}\right)^{s} \frac{\partial H}{\partial\left(\partial_{x}^{s} u_{j}\right)}$ or in turn

$$
\sum_{k=0}^{n-1} \xi_{k} \sum_{i=k}^{n-1} h_{k-i}=\sum_{k=0}^{n-1} \frac{\delta h}{\delta u_{k}} \xi_{k}
$$

which gives

$$
h_{0}=\frac{\delta h}{\delta u_{n-1}}, \quad h_{i}=\frac{\delta h}{\delta u_{n-i-1}}-\frac{\delta h}{\delta u_{n-i}} .
$$

From formulas (4.2) one can easily compute

$$
\begin{aligned}
h_{2 s+1}= & \sum_{i=0}^{s} \frac{3 \alpha}{2} \Lambda^{-1}\left(\Lambda^{-1}\left(u_{i}\right) \Lambda^{-1}\left(u_{2 s+1-i}\right)+\frac{1}{2} \sum_{i=0}^{s} \Lambda^{-1}\left(u_{i}\right) \Lambda^{-1}\left(u_{2 s+1-i}\right)\right. \\
& +\frac{1}{4} \sum_{i=0}^{2 s+1} \Lambda^{-1}\left(\Lambda^{-1}\left(u_{i}\right) u_{2 s+1-i}\right)+a \Lambda^{-2} \partial_{x}^{2} u_{2 s+1} \\
h_{2 s}=\quad & \sum_{i=0}^{s-1} \frac{3 \alpha}{2} \Lambda^{-1}\left(\Lambda^{-1}\left(u_{i}\right) \Lambda^{-1}\left(u_{2 s-i}\right)+\frac{3 \alpha}{4} \Lambda^{-1}\left(\Lambda^{-1}\left(u_{s}\right)\right)^{2}\right. \\
& +\frac{1}{2} \sum_{i=0}^{s-1} \Lambda^{-1}\left(u_{i}\right) \Lambda^{-1}\left(u_{2 s-i}\right)+\frac{1}{4}\left(\Lambda^{-1}\left(u_{s}\right)\right)^{2} \\
& +\frac{1}{4} \sum_{i=0}^{2 s} \Lambda^{-1}\left(\Lambda^{-1}\left(u_{i}\right) u_{2 s-i}\right)+a \Lambda^{-2} \partial_{x}^{2} u_{2 s}
\end{aligned}
$$

Now the frozen tensor $P$ at the point $u_{0}=\frac{1}{2}, u_{i}=0$ for $i=1, \ldots, n-1$ is the diagonal $n \times n$ matrix:

$$
P_{f}=\left(\begin{array}{ccccc}
\Lambda & 0 & 0 & \ldots & 0 \\
0 & \Lambda & 0 & \ldots & 0 \\
0 & 0 & \Lambda & \ldots & 0 \\
\vdots & \vdots & \vdots & \ddots & 0 \\
0 & 0 & 0 & \ldots & \Lambda
\end{array}\right)
$$


therefore applying it to $\left(h_{0}, \ldots, h_{n-1}\right)$ we have

$$
(P(\underline{h}))_{i}=\Lambda \partial_{x} h_{i} \quad \forall i=0 \ldots n-1
$$

Performing the same computations of [6] page 132 we obtain that

$$
\Lambda \partial_{t} v_{r}=\Lambda \partial_{x} h_{r}=\sum_{i=0}^{r} \frac{3 \alpha}{4} \partial_{x}\left(v_{i} v_{r-i}\right)+\frac{1}{4} \sum_{i=0}^{r} \Lambda \partial_{x}\left(v_{i} v_{r-i}\right)+\frac{1}{4} \sum_{i=0}^{r} \partial_{x}\left(v_{i} u_{r-i}\right)+a \partial_{x}^{3} v_{r}
$$

where $v_{i}=\Lambda^{-1} u_{i}$ for $i=0, \ldots, n-1$. Recalling that $\Lambda=\alpha-\beta \partial_{x}^{2}$ and that $c_{i}=\delta_{0 i}$ $(i=0 \ldots n)$ we obtain the equations $(3.9)$.

The obtained equations are exactly, in the $\mathrm{KdV}$ case, the polynomial equations of [1] relatives to the polynomial algebras $\mathfrak{s l}_{2}^{(n)}(\mathbb{C})$. Therefore it is natural to call "n-polynomial" the previous bihamiltonian system also in the Camassa-Holm case. The description of the bihamiltonian structure of the "polynomial Hunter-Saxon" i.e., of the equations (3.9) in the case when $\alpha=0$ turns out to be a little more complicate. Indeed, exactly as in the case with $n=1$ treated by Khesin and Misiołek [6], problems arise because in this setting the operator $\Lambda$ and the inertia operator (3.4) as well fails to be invertible. The key point is to observe that on the space $v_{i r}^{(n)}$ naturally acts the group $S_{n}$ given by the direct product of $n$ copies of the group $S^{1}$ as

$$
\begin{aligned}
K_{n} \times \operatorname{vir}^{(n)} & \rightarrow \operatorname{vir}^{(n)} \\
\left(\left(p_{0}, \ldots, p_{n}\right),\left(u_{0}(x), \ldots, u_{n}(x)\right)\right) & \mapsto\left(u_{0}\left(x+p_{0}\right), \ldots, u_{n}\left(x+p_{n}\right)\right)
\end{aligned}
$$

Theorem 4.2. The equations (3.9) with $\alpha=0$ are bihamiltonian on the quotient space vir $^{(n)} / S_{n}$ with Hamiltonian:

$$
H=\int_{S^{1}} \sum_{l=0}^{n}\left(\sum_{\substack{i, j, k=0 \\ i+j+k=n-1}}^{l} \frac{1}{4} \Lambda^{-1}\left(u_{i}\right) \Lambda^{-1}\left(u_{j}\right) u_{k}\right) d x=\int_{S^{1}} h d x
$$

where $\Lambda=-\beta \partial_{x}^{2}$ and the second Poisson structure is given by freezing the canonical one at the point $u_{j}=0 j=0, \ldots n, c_{i}=0 i=0, \ldots n-1$, and $c_{n}=\beta$.

Proof. Since the actually computations are the same of those of the previous theorem, it remains only to check that the Hamiltonian is well defined on the space $\operatorname{vir}^{(n)} / S_{n}$. But this latter claim follows almost immediately from the observation that $\Lambda$ is invertible on this space.

A slightly generalization of the diagonal case exists at least in the case of 2 coupled equations. To perform the computation we need the following technical restriction:

$$
\frac{\alpha_{0}}{\alpha_{1}}=\frac{\beta_{0}}{\beta_{1}}=\gamma
$$

Let us consider now the Hamiltonian density:

$$
\begin{aligned}
h= & \frac{\alpha_{1}}{4}\left(\Lambda^{-1} u\right)_{0}^{3}+\frac{1}{4}\left(\Lambda^{-1} u\right)_{0}^{2} u_{0}+\frac{a_{1}}{2}\left(\Lambda^{-1} u_{x}\right)_{0}^{2} \\
& +\frac{3 \alpha_{1}}{4}\left(\Lambda^{-1} u\right)_{0}^{2}\left(\Lambda^{-1} u\right)_{1}+\frac{1}{4}\left(\Lambda^{-1} u\right)_{0}^{2} u_{1}+\frac{1}{2}\left(\Lambda^{-1} u\right)_{0}^{2}\left(\Lambda^{-1} u\right)_{1} u_{0} \\
& +a_{1}\left(\Lambda^{-1} u_{x}\right)_{0}\left(\Lambda^{-1} u_{x}\right)_{1}+\frac{a_{0}}{2}\left(\Lambda^{-1} u_{x}\right)_{0}^{2}+\frac{2 \alpha_{0}-\alpha_{1}}{4}\left(\Lambda^{-1} u\right)_{0}^{3}
\end{aligned}
$$


where $\Lambda$ indicates the inertia operator (3.4) in this particular case:

$$
\Lambda:=\left(\begin{array}{cc}
\Lambda_{1} & 0 \\
\Lambda_{0}-\Lambda_{1} & \Lambda_{1}
\end{array}\right)=\left(\begin{array}{cc}
\alpha_{1}-\beta_{1} \partial_{x}^{2} & 0 \\
\alpha_{0}-\beta_{0} \partial_{x}^{2}-\left(\alpha_{1}-\beta_{1} \partial_{x}^{2}\right) & \alpha_{1}-\beta_{1} \partial_{x}^{2}
\end{array}\right)
$$

and

$$
\begin{gathered}
v_{0}=\left(\Lambda^{-1} u\right)_{0}=\Lambda_{1}^{-1} u_{0} \\
v_{1}=\left(\Lambda^{-1} u\right)_{1}=\Lambda_{1}^{-1} u_{1}-\left(\Lambda_{0}-\Lambda_{1}\right) \Lambda_{1}^{-2} u_{0} .
\end{gathered}
$$

where we used explicitly the commutativity of the operators $\Lambda_{0}$ and $\Lambda_{1}$ for a good definition of the inverse of $\Lambda$. From (4.2) we obtain for the gradient components:

$$
\begin{aligned}
h_{0}=\frac{\delta h}{u_{1}}= & \frac{3 \alpha_{1}}{4} \Lambda_{1}^{-1} v_{0}^{2}+\frac{1}{4}\left(\Lambda_{0}-\Lambda_{1}\right) \Lambda_{1}^{-2} v_{0}^{2}+\frac{1}{2} \Lambda_{1}^{-1}\left(v_{0} \Lambda_{1} v_{0}\right)-a_{1} \Lambda^{-1} \partial_{x}^{2} v_{0} \\
h_{1}=\frac{\delta h}{u_{0}}-\frac{\delta h}{u_{1}}= & \frac{3 \alpha_{1}}{2} \Lambda_{1}^{-1}\left(v_{0} v_{1}\right)-\frac{3 \alpha_{1}}{2} v_{0}^{2}+\frac{1}{2} \Lambda_{1}^{-1}\left(v_{0} u_{1}\right)+\frac{1}{2} v_{0} v_{1} \\
& +\frac{1}{2} \Lambda_{1}^{-1}\left(v_{1} \Lambda_{1} v_{0}\right)-\frac{1}{2}\left(\Lambda_{0}-\Lambda_{1}\right) \Lambda_{1}^{-2}\left(v_{0} \Lambda_{1} v_{0}\right)-a_{1} \Lambda_{1}^{-1} \partial_{x}^{2} v_{1} \\
& -\left(\Lambda_{0}-\Lambda_{1}\right) \Lambda_{1}^{-2} \partial_{x}^{2} v_{0}-a_{0} \Lambda_{1}^{-1} \partial_{x}^{2} v_{0}+\frac{6 \alpha_{0}-3 \alpha_{1}}{4} \Lambda_{1}^{-1}\left(v_{0}^{2}\right) .
\end{aligned}
$$

Using the same freezing points as in the diagonal case, i.e. $u_{0}=\frac{1}{2}, u_{1}=0, c_{0}=\beta_{1}=a_{0}$ and $c_{1}=\beta_{0}-\beta_{1}=a_{1}$, the Poisson structure becomes equal to $\Lambda \partial_{x}$ and the equations in the case (4.5) are:

$$
\begin{aligned}
\Lambda_{1} u_{0} & =\Lambda_{1} \partial_{x} h_{0} \\
\left(\Lambda_{0}-\Lambda_{1}\right) u_{0}+\Lambda_{1} u_{1} & =\left(\Lambda_{0}-\Lambda_{1}\right) \partial_{x} h_{0}+\Lambda_{1} \partial_{x} h_{1}
\end{aligned}
$$

or

$$
\left\{\begin{array}{l}
\alpha_{1} \partial_{t} u_{0}-\beta_{1} \partial_{t} \partial_{x}^{2} u_{0}=3 \alpha_{1}\left(\partial_{x} u_{0}\right) u_{0}-2 \beta_{1}\left(\partial_{x}^{2} u_{0}\right) \partial_{x} u_{0}-\beta_{1}\left(\partial_{x}^{3} u_{0}\right) u_{0}-c_{0} \partial_{x}^{3} u_{0} \\
\alpha_{0} \partial_{t} u_{0}-\beta_{0}\left(\partial_{t} \partial_{x}^{2} u_{0}\right)-\left(\alpha_{1} \partial_{t} u_{0}-\beta_{1} \partial_{t} \partial_{x}^{2} u_{0}\right)+\alpha_{1} \partial_{t} u_{1}-\beta_{1} \partial_{t} \partial_{x}^{2} u_{1} \\
=3\left(\alpha_{0}-\alpha_{1}\right)\left(\partial_{x} u_{0}\right) u_{0}-2\left(\beta_{0}-\beta_{1}\right)\left(\partial_{x}^{2} u_{0}\right) \partial_{x} u_{0}-\left(\beta_{0}-\beta_{1}\right)\left(\partial_{x}^{3} u_{0}\right) u_{0}-c_{1} \partial_{x}^{3} u_{0}+ \\
+3 \alpha_{1} \partial_{x}\left(u_{0} u_{1}\right)-2 \beta_{1} \partial_{x}\left(\partial_{x}\left(u_{0 x}\right) \partial_{x} u_{1}\right)-\beta_{1}\left(\partial_{x}^{3} u_{1}\right) u_{0}-\beta_{1}\left(\partial_{x}^{3} u_{0}\right) u_{1}-c_{0} \partial_{x}^{3} u_{1}
\end{array}\right.
$$

which, after some algebraic manipulations, becomes (3.10) as expected.

Acknowledgments. The authors would like to thank the referee for his interesting and useful suggestions and remarks on the first version of this paper which have improved it.

\section{References}

[1] Casati P and Ortenzi G, New Integrable Hierarchies from Vertex Operator Representations of Polynomial Lie Algebras, J. Geom. Phys. 56 (2006), 418-449, arXiv:nlin.SI/0405040.

[2] Camassa R and Holm D D, An integrable shallow water equation with peaked solitons, Phys. Rev. Lett. 71 (1993), 1661-1664.

[3] Fuks D B, Cohomology of infinite-dimensional Lie algebras, Contemporary Soviet mathematics, New York Consultants Bureau, (1986), 339. 
[4] Hunter J K and Saxton R, Dynamics of director fields, SIAM J. Appl. Math. 51 (1991), 1498-1521.

[5] KAC V G, Infinite dimensional Lie algebras, 3rd ed., Cambridge University press, Cambridge, 1990, pp. xix +400 .

[6] Khesin B and Misiołek G, Euler equations on homogeneous spaces and Virasoro orbits, Adv. Math. 176 (2003), 116-144.

[7] Magnano G and Magri F, Poisson-Nijenhuis structures and Sato hierarchy, Rev. Math. Phys. 3 (1991), 403-466.

[8] Pressley A and Segal G, Loop Groups, Oxford University, Oxford, 1986, 316.

[9] Eswara Rao S and Moody R V, Vertex Representations for N-Toroidal Lie Algebras and a Generalization of the Virasoro Algebra, Commun. Math. Phys. 159 (1994), 239264 .

[10] Sakovich S Yu, Coupled KdV equations of Hirota-Satsuma type, J. Nonlinear Math. Phys. 6 (1999), 255-262.

[11] Segal G and Wilson G, Loop Groups and equations of the KdV type, Publ. Math.Paris 61 (1985), 5-65. 